\title{
ARTICLE
}

\section{Substrate-decoupled, bulk-acoustic wave gyroscopes: Design and evaluation of next-generation environmentally robust} devices

\author{
Diego E. Serrano ${ }^{1}$, Mohammad F. Zaman ${ }^{1}$, Amir Rahafrooz ${ }^{1}$, Peter Hrudey ${ }^{1}$, Ron Lipka ${ }^{1}$, Duane Younkin ${ }^{1}$, Shin Nagpal ${ }^{1}$, ljaz Jafri ${ }^{1}$ and
} Farrokh Ayazi $^{1,2}$

This paper reports on a new type of high-frequency mode-matched gyroscope with significantly reduced dependencies on environmental stimuli such as temperature, vibration, and shock. A novel stress-isolation system is used to effectively decouple an axis-symmetric bulk-acoustic wave (BAW) vibratory gyro from its substrate, minimizing the effect that external sources of error have on the offset and scale factor of the device. Substrate-decoupled (SD) BAW gyros with a resonance frequency of $4.3 \mathrm{MHz}$ and $Q$ values near 60000 were implemented using the high aspect ratio poly and single-crystal silicon (HARPSS) process to achieve ultra-narrow capacitive gaps. Wafer-level packaged sensors were interfaced with a customized application-specific integrated circuit (ASIC) to achieve low variations in the offset across temperature $\left( \pm 26^{\circ} \mathrm{s}^{-1}\right.$ from -40 to $\left.85^{\circ} \mathrm{C}\right)$, supreme random-vibration immunity $\left(0.012^{\circ} \mathrm{s}^{-1} \mathrm{~g}_{\mathrm{RMS}}^{-1}\right)$ and excellent shock rejection. With a scale factor of $800 \mu \mathrm{V}\left({ }^{\circ} \mathrm{s}^{-1}\right)^{-1}$, the SD-BAW gyro system attains a large full-scale range $\left( \pm 1250^{\circ} \mathrm{s}^{-1}\right)$ with a non-linearity of less than $0.07 \%$. A measured angle-random walk (ARW) of $0.39^{\circ} / \sqrt{ } \mathrm{h}$ and a bias instability of $10.5^{\circ} \mathrm{h}^{-1}$ are dominated by the thermal and flicker noise of the integrated circuit (IC), respectively. Additional measurements using external electronics show bias-instability values as low as $3.5^{\circ} \mathrm{h}^{-1}$, which are limited by feed-through signals coupled from the drive loop to the sense channel, which can be further reduced through proper re-routing of the gyroscope pin-out configuration.

Keywords: Bulk-acoustic wave; environmental stability; MEMS gyroscope; support loss; vibration rejection

Microsystems \& Nanoengineering (2016) 2, 16015; doi:10.1038/micronano.2016.15; Published online: 25 April 2016

\section{INTRODUCTION}

Micromachined gyroscopes have enabled a myriad of applications that range from basic motion detection for gaming to safety control systems in automobiles ${ }^{1}$. More recently, an increased interest in the use of microelectromechanical system inertial sensors for dead reckoning and pedestrian navigation in handheld electronics has placed stringent requirements on the die size, power consumption, and overall performance of this type of device. To date, most commercially available rate sensors have been designed as low-frequency flexural tuning-fork gyroscopes (TFGs), which are typically sensitive to random vibrations and prone to linear accelerations, such as those experienced under shock. These limitations complicate the use of TFG technology in large-volume, high-end applications, particularly in personal navigation, for which dependencies on fluctuations in the environment translate into long-term drift in the output of the system. Additionally, in recent months, concerns about the high sensitivity of consumer-grade gyroscopes in response to lowfrequency pressure signals that can be used to recover audio have increased as a potential threat for eavesdropping ${ }^{2}$, justifying the need for more environmentally robust rotation sensors.

Acceleration suppression mechanisms can be implemented in TFGs to alleviate part of this problem using redundant proof masses that reject shock and vibration as common-mode signals ${ }^{3}$.
However, this compensation technique results in a significant increase in the size of these devices and could require electromechanical calibration to compensate for fabrication imperfections ${ }^{4}$, making them more suitable in low-volume applications.

As an alternative, the degenerate modes of bulk-acoustic wave (BAW) resonators can be used to implement axis-symmetric mode-matched gyroscopes that operate in the $\mathrm{MHz}$ range with high quality factors at moderate vacuum levels (that is, 1 to 10 Torr $)^{5}$. Given their high-frequency nature, BAW gyros inherently reject the effects of random vibrations in the environment and are highly immune to shock.

However, like in any other type of gyroscope, differences in the loss mechanisms of the two degenerate modes can lead to damping coupling, which results in unwanted environmentally dependent offset variations ${ }^{6}$. In axis-symmetric gyros, fabrication or material imperfections can cause different support-loss rates for each of the modes, particularly if the devices are implemented in anisotropic substrates such as (100) single-crystal silicon (SCS). Therefore, to minimize the effects of damping mismatch, this paper presents a high-frequency gyro for $z$-axis rate detection that combines the properties of a BAW sensor with an isolation structure that significantly reduces anchor loss in the system. The substrate-decoupled (SD) BAW gyro thus attains a markedly improved environmental performance and offers the versatility 
necessary for high-volume production for consumer, automotive and industrial applications.

\section{DESIGN AND SIMULATION}

A schematic diagram of the SD-BAW gyroscope is shown in Figure 1. The sensor consists of an active resonator region anchored through a stress isolation system, which is designed to effectively decouple the gyro from its substrate at the resonance frequency of the modes of vibration. Electrodes with ultra-narrow capacitive gaps $(270 \mathrm{~nm})$ surround the structure to allow electrostatic excitation, readout, and frequency tuning ${ }^{7}$.

\section{Basic principles of operation}

Similar to ring- and shell-type gyroscopes ${ }^{8}$, SD-BAW gyros are axissymmetric devices that use the degenerate modes of a vibrating structure to detect rotation ${ }^{5}$. Therefore, the behavior of an SDBAW device is described by two orthogonal, second-order systems coupled to each other by a Coriolis force that is proportional to an applied angular velocity:

$$
\begin{gathered}
m_{11} \ddot{q}_{1}(t)+b_{11} \dot{q}_{1}(t)+b_{12} \dot{q}_{2}(t)+k_{11} q_{1}(t)+k_{12} q_{2}(t) \\
=\sum_{i=1}^{k} F_{1, i}-2 \lambda m_{22} \Omega(t) \dot{q}_{2}(t) \\
m_{22} \ddot{q}_{2}(t)+b_{22} \dot{q}_{2}(t)+b_{21} \dot{q}_{1}(t)+k_{22} q_{2}(t)+k_{21} q_{1}(t) \\
=\sum_{i=1}^{k} F_{2, i}+2 \lambda m_{11} \Omega(t) \dot{q}_{1}(t)
\end{gathered}
$$

In Equations (1a) and (1b), $\Omega(t)$ corresponds to the rate of rotation applied around an axis normal to the plane of modal vibration. The factor $\lambda$ is known as the angular gain of the gyroscope, which is dictated by the Bryan effect ${ }^{9}$; its value is determined by the device's geometry and mode shape. The terms $m_{11}, k_{11}$, and $b_{11}$ are the effective mass, effective stiffness, and damping associated with mode 1 , respectively; $m_{22}, k_{22}$, and $b_{22}$ correspond to these same parameters of mode 2 . In a perfect axis-symmetric gyroscope, the properties of both modes are identical, making $m_{11}=m_{22}, b_{11}=b_{22}, k_{11}=k_{22}$, and thus their resonances frequencies equal $\left(\omega_{01}=\omega_{02}\right)$. However, material and fabrication imperfections lead to differences in the elastic and/or inertial characteristics of the degenerate modes, resulting a frequency split between them. Additionally, in an ideal vibratory gyro, the two modes should only be coupled to each other by the Coriolis forces $-2 m_{22} \lambda \dot{q}_{2}(t) \Omega(t)$ and $+2 m_{11} \lambda \dot{q}_{1}(t) \Omega(t)$; however, structural imperfections in a real device produce undesired mode-to-mode coupling ${ }^{10}$. These effects are captured in Equations (1a) and (1b) via the stiffness-coupling terms $k_{12}$ and $k_{21}$ and the dampingcoupling coefficients $b_{12}$ and $b_{21}$.
The variables $F_{1, i}$ and $F_{2, j}$ correspond to the $k$ and $/$ electrostatic forces acting on modes 1 and 2, respectively, which are used to drive and control the mode of operation of the gyroscope and to compensate for the aforementioned elastic and inertial imperfections ${ }^{11}$.

Lastly, the parameters $q_{1}(t)$ and $q_{2}(t)$ in Equations (1a) and (1b) are the generalized coordinates of modes 1 and 2, respectively, that determine the gyroscope's modal displacements in combination with the mode-shape functions ${ }^{12}$. Figure 2 shows the simulated modal deformations of the second-elliptical $(n=3)$ in-plane degenerate modes of an SD-BAW gyro that was created in a 40- $\mu$ m-thick (100) SCS silicon-on-insulator (SOI) wafer with a radius of $420 \mu \mathrm{m}$. (100) SCS substrates were selected instead of (111) SCS substrates because the $n=3$ modes maintain degeneracy, and their resonance frequencies are less sensitive to crystalline misalignment ${ }^{13}$.

When configured as a rotation-rate sensor, one of the two degenerate modes of the gyroscope (that is, the drive mode) is excited into self-oscillation to establish a well-regulated displacement reference (that is, $q_{1}(t)=\bar{q}_{1} \sin \left(\omega_{\text {drive }} t\right)$, where $\bar{q}_{1}$ is constant, and $\omega_{\text {drive }}=\omega_{01}$ ideally). In the presence of an angular velocity $\Omega(t)$, the second mode (that is, the sense mode) is excited by the generated Coriolis force, causing a displacement that can be read in the form of a current through a capacitive electrode aligned with the antinodes of the sense mode. If the frequency of the established drive-mode oscillation is equal to the resonance frequency of the sense mode (that is, $\omega_{\text {drive }}=\omega_{02}$ ), the generated displacement due to an applied rotation rate can be described by

$$
q_{2 \text { corilis }}(t)=\frac{2 \lambda \mathcal{Q}_{2}}{\omega_{02}} \Omega(t) \bar{q}_{1} \sin \left(\omega_{02} t\right)
$$

where $\mathcal{Q}_{2}=b_{22} / \sqrt{ }\left(k_{22} m_{22}\right)$ is the quality factor of the sense mode. Therefore, $q_{2 \text { coriolis }}(t)$ is an amplitude-modulated signal with an envelope proportional to the rate of rotation $\Omega(t)$ and carrier frequency and phase equal to those of the drive-loop displacement.

\section{Compensation of anisoinertia and anisoelasticity}

Material and fabrication imperfections typically introduce differences in the masses (anisoinertia) and stiffnesses (anisoelasticity) of the two degenerate modes ${ }^{10}$. These effects translate into a frequency split that affects the overall performance of the gyro ${ }^{14}$. Additionally, these non-idealities tend to generate mode-to-mode coupling terms $\left(k_{12}\right.$ and $\left.k_{21}\right)$ that translate into a sense-mode zerorate output (ZRO) displacement error signal in an angular-rate sensor:

$$
q_{2 \text { stiffness }}(t)=\frac{\mathcal{Q}_{2}}{m_{22} \omega_{02}^{2}} k_{21} \bar{q}_{1} \cos \left(\omega_{02} t\right)
$$

Although $q_{2 \text { stiffness }}(t)$ is detrimental to gyro performance, the fact that its phase is $90^{\circ}$ with respect to $q_{2 \text { coriolis }}(t)$ makes it easy to
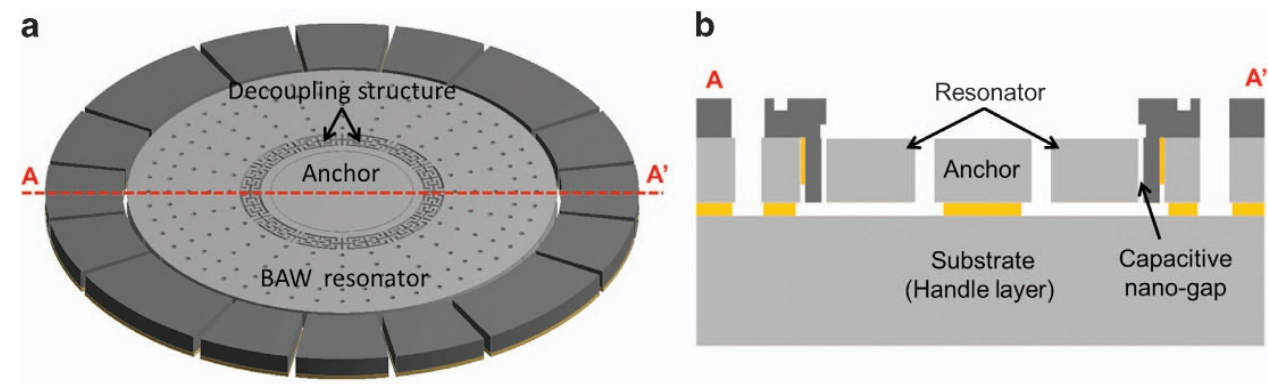

Figure 1 (a) Schematic view of the SD-BAW gyroscope. An active resonating gyro region is effectively decoupled from the substrate by a stress decoupling structure. (b) Electrodes with ultra-narrow capacitive gaps $(270 \mathrm{~nm})$ created with the HARPSS process are used for electrostatic actuation and readout. HARPSS, high aspect-ratio poly and single-crystal silicon; BAW, bulk-acoustic wave; SD, substrate-decoupled. 

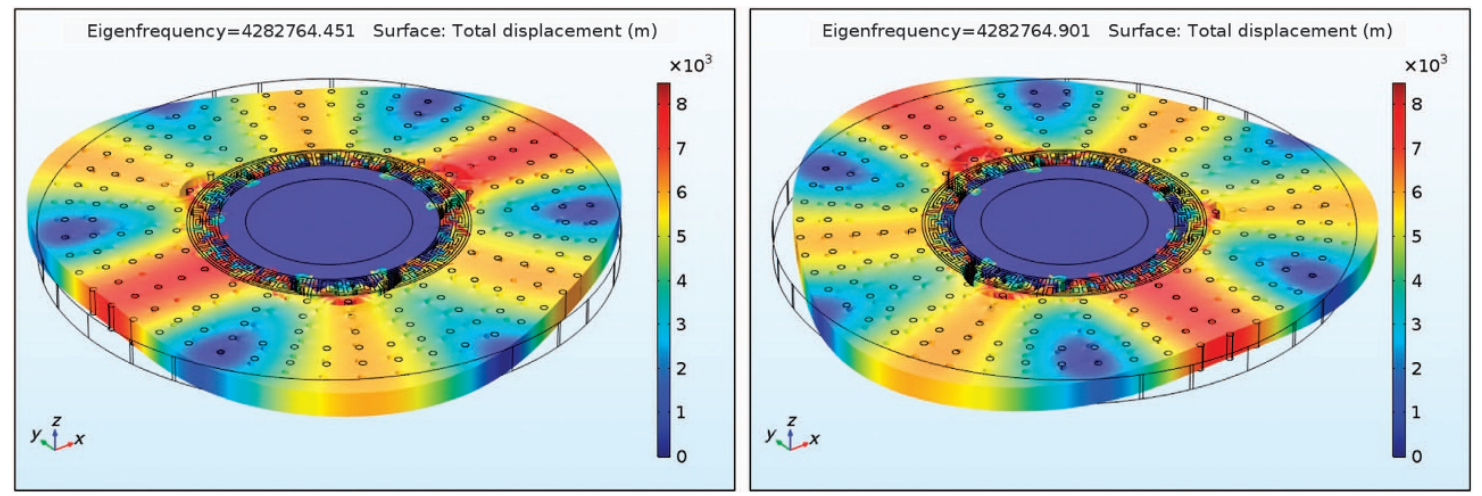

Figure 2 Modal simulation response of the second elliptical $(n=3)$ in-plane modes of an SD-BAW gyroscope with $420 \mu \mathrm{m}$ radius, created on a $40-\mu \mathrm{m}$-thick (100) SCS substrate (electrodes not shown; total displacement normalized with respect to mass matrix). BAW, bulk-acoustic wave; SCS, single-crystal silicon; SD, substrate-decoupled.
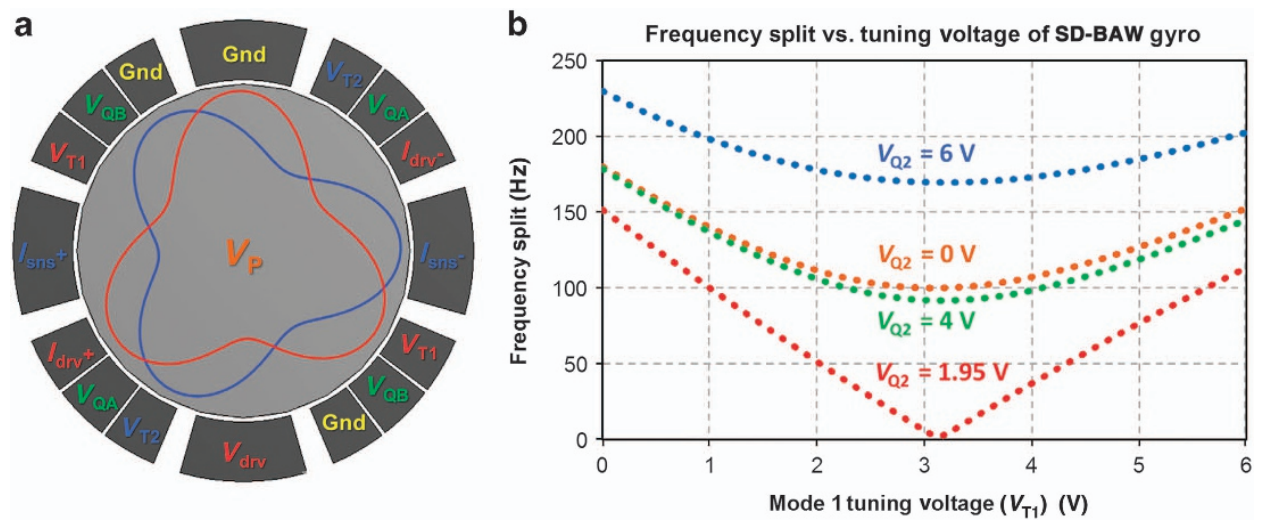

Figure 3 (a) Electrode configuration for the SD-BAW gyroscope. (b) Simulated electrostatic frequency-tuning and stiffness-decoupling for an imperfect (ellipsoidal) SD-BAW gyroscope. Mode decoupling was achieved with $V_{\mathrm{Q} 2}=1.95 \mathrm{~V}$, and mode matching was achieved at $V_{\mathrm{T} 1}=3.15 \mathrm{~V}$. The resonator was biased at $V_{\mathrm{P}}=18 \mathrm{~V}$ with all other electrodes at a common-mode voltage of $2.5 \mathrm{~V}$. The residual split was less than $0.05 \mathrm{~Hz}$ using a voltage resolution of $10 \mathrm{mV}$. BAW, bulk-acoustic wave; SD, substrate-decoupled.

identify and correct, particularly when using coherent synchronous demodulation ${ }^{15}$.

To compensate for this frequency split and the ZRO errors caused by the described anisoelasticity and anisoinertia, electrostatic spring softening can be used. Electrodes aligned with the antinodes of the degenerate modes of interest will introduce a direct stiffness term. Conversely, electrodes located between antinodes will affect the overall indirect stiffness coupling, allowing quadrature cancellation ${ }^{10}$.

Figure $3 a$ shows the electrode configuration used for the SD-BAW gyroscope presented in this study. Electrodes $V_{\mathrm{T} 1}$ and $V_{\mathrm{T} 2}$ are used to tune the resonances of modes 1 and 2, respectively, to compensate for the frequency splits. Electrodes $V_{\mathrm{QA}}$ and $V_{\mathrm{QB}}$ are used to cancel out the mode-coupling terms. The pads labeled $I_{\mathrm{drv}}+, I_{\mathrm{drv}}-, I_{\mathrm{sns}}+, I_{\mathrm{sns}}-$ correspond to the positive and negative readout electrodes for the drive and sense modes, and $V_{\mathrm{drv}}$ corresponds to the drive excitation electrode. The resonating gyro structure is biased at a polarization voltage $V_{P}$; the electrodes labeled 'Gnd' are inactive in this study but can be used for additional frequency tuning, signal readout and/or modal excitation.

To verify the effectiveness of the tuning and decoupling electrodes, electromechanical FEA simulations were performed on an imperfect SD-BAW gyroscope. In the model, the outer boundary of the resonator was built as an ellipse rather than a perfect circle to capture the effects of lens astigmatism that can be produced during fabrication in the lithography step that defines the structure. The difference between the ellipse semiaxes was obtained empirically from previous generations of BAW resonators. Figure $3 \mathrm{~b}$ shows an example of the frequency-tuning and mode-decoupling response when $V_{\mathrm{T} 1}$ and $V_{\mathrm{O} 2}$ are varied. Only when the modes are fully decoupled can a frequency split of $0 \mathrm{~Hz}$ be achieved ${ }^{10}$.

\section{Mechanical reduction of anisodamping}

The damping terms $b_{11}$ and $b_{22}$ in Equations (1a) and (1b) are a measure of the amount of vibration energy lost by each of the two modes typically to the surrounding environment ${ }^{16,17}$. If the sources of damping are not aligned with the antinodes of the modes of interest, each mode will also experience a coupling force proportional to the vibration velocity of its degenerate pair ${ }^{18}$. This effect is captured by the terms $b_{12}$ and $b_{21}$, which will generate an undesired sense displacement ZRO in a rotation-rate sensor:

$$
q_{2 \text { damping }}(t)=-\frac{\mathcal{Q}_{2}}{m_{22} \omega_{02}} b_{21} \bar{q}_{1} \sin \left(\omega_{02} t\right)
$$

By comparing Equations (2), (3), and (4), it can be concluded that unlike $q_{2 \text { stiffness }}(t)$, the error signal $q_{2 \text { damping }}(t)$ is in phase with $q_{\text {2coriolis }}(t)$, which makes them indistinguishable from each other ${ }^{19}$. Complex circuit architectures can be used to reject $q_{2 \text { damping }}(t)$ as a 

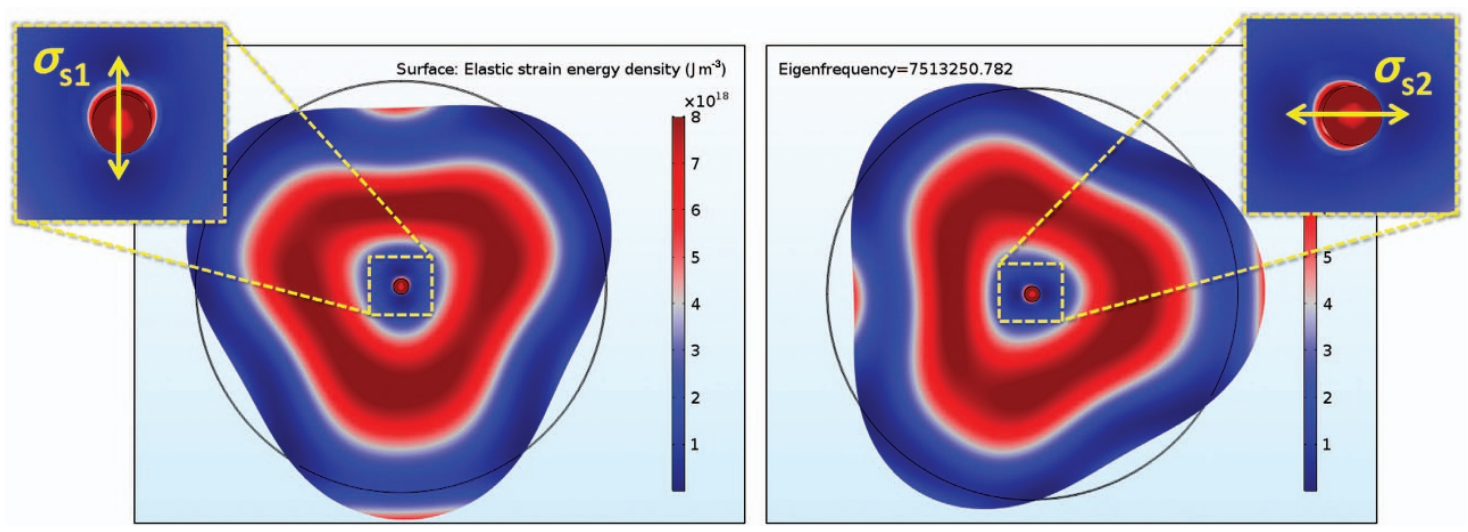

Figure 4 SED distribution of the second elliptical in-plane mode of a center-supported disk BAW resonator in (100) SCS. The anisotropic nature of the material causes a directionally dependent shear stress in the resonator-substrate boundary that is different for the two modes. (Red regions indicate where the SED is maximum; blue regions indicate where the SED is minimum.) BAW, bulk-acoustic wave; SCS, single-crystal silicon; SD, substrate-decoupled; SED, strain energy density.

common-mode signal using mode reversal ${ }^{20}$. However, in a rate gyroscope system, this approach results in either extremely large settling times to achieve alternating modal excitations (limiting the effective bandwidth of the system) ${ }^{21}$ or high power consumption to drive both modes simultaneously. Therefore, it is critical to guarantee that the damping mechanisms that affect the two modes are designed to be as symmetric as possible.

In a resonant system, the effects of damping are typically expressed in terms of its quality factor, which quantifies the ratio of the peak energy stored in the resonator $W$ to the energy dissipated per cycle $\Delta W$ :

$$
\mathcal{Q}=2 \pi \frac{W}{\Delta W}
$$

The total value of $\mathcal{Q}$ is then determined by the contributions of all different sources of energy loss that affect the system, which include squeeze-film damping (SFD) ${ }^{22}$, thermoelastic damping (TED) ${ }^{23}$, support or anchor loss ${ }^{24}$, and other surface ${ }^{25}$ and intrinsic losses ${ }^{16}$, which are typically minimal in SD-BAW gyros. Given the highfrequency nature of SD-BAW gyros, SFD typically has minor contributions to the total $\mathcal{Q}$, even at moderate pressure levels (for example, 1 to 10 Torr). Additionally, even when operated at higher pressures, the axis-symmetric nature of the gyro causes the SFD values of both modes to be identical, thus not contributing to damping coupling. Similarly, TED in SD-BAW gyros is determined by the localized compressive and extensive strain generated around the release holes in the resonator. The hole-pattern arrangement can then be designed to be axis-symmetric and affect both modes identically, minimizing the contribution of TED to the coupling terms.

Conversely, the value of the anchor loss of each of the two degenerate modes is highly dependent on how the resonator couples to its substrate. This occurs because, even though the maximum energy stored on each mode is a function of controllable parameters such as material properties and geometry, the energy dissipated per cycle depends on the integral of the strain energy density (SED) at the resonator - substrate interface ${ }^{24}$ :

$$
\Delta W=\pi \int_{V_{s}} \sigma_{s} \epsilon_{s} \mathrm{~d} V
$$

In Equation (6), $V s$ is the volume of the support region, and $\sigma_{s}$ and $\varepsilon_{s}$ are the strain and stress at the resonator-substrate interface, respectively. A lower-bound 'worst-case' value of $\mathcal{Q}$ due to anchor loss ( $\left.\mathcal{Q}_{\text {anchor }}\right)$ can be estimated for each of the modes by assuming that all the strain energy at the interface is dissipated into the substrate (that is, there are no acoustic reflections) ${ }^{26,27}$. However, this condition is only valid when the dimensions of the substrate are much larger than the wavelength of the acoustic wave generated by the resonator, which is rarely true. For example, the wavelength of the acoustic signal generated by a 4-MHz resonator traveling through silicon is approximately $2 \mathrm{~mm}$, which would require the substrate to be much larger than this value in all directions to assume that no energy is reflected. Therefore, the real effective $\mathcal{Q}_{\text {anchor }}$ will be a function of how the energy propagates within the substrate, which can change dramatically in the presence of substrate spurious modes with frequencies that are strongly dependent on how the device is singulated, mounted, and attached to its surroundings. More importantly, the way the energy dissipates and reflects for each of the two degenerate modes of an axis-symmetric gyro can differ significantly, causing an excessive amount of damping coupling. For example, Figure 4 shows the SED distribution of the second-elliptical modes in a center-supported BAW disk resonator in (100) SCS. Due to the anisotropic nature of the device material, an effective shear stress is generated at the resonator/substrate interface that aligns with the antinode with the largest deformation of each of the modes. This will cause a loss mechanism that will propagate in different directions for each of the two modes and that depends on how the energy interacts with all spurious modes in the substrate.

Consequently, when a resonator is tightly coupled to its substrate, it would be difficult to estimate the effective anchor losses of each of the two modes; it would be even more complex to quantify their contribution to the undesired error signal $q_{\text {2damping }}(t)$.

A practical solution to this problem is to implement an effective acoustic isolation mechanism between the resonator structure and its surroundings. Several techniques to minimize anchor loss in microresonators have been reported in the literature, including the use of acoustic stop-band phononic crystal structures ${ }^{28}$, the addition of half-wavelength acoustic reflectors ${ }^{29}$, or the exploitation of the mismatch of material properties for acoustic isolation ${ }^{30}$. However, a less intricate way to isolate a resonant structure is including a stress isolation structure ${ }^{31}$. To reduce the final stress imparted to the anchoring point, the isolation apparatus should be capable of effectively attenuating the strain produced by the deformation in the resonator. Figure 5 shows a close-up view of the SED distribution in the stress isolation system used for an SDBAW gyroscope. It is clearly shown that the decoupling structure attenuates the strain induced on the center anchor, providing excellent isolation between the resonator and its substrate.

Part of the energy that would be originally dissipated into the substrate will now be stored within the resonator; however, another portion of it will be dissipated in the form of heat generated within the new compressive and tensile regions in the decoupling system (that is, TED within the stress isolation structure). If the stress 

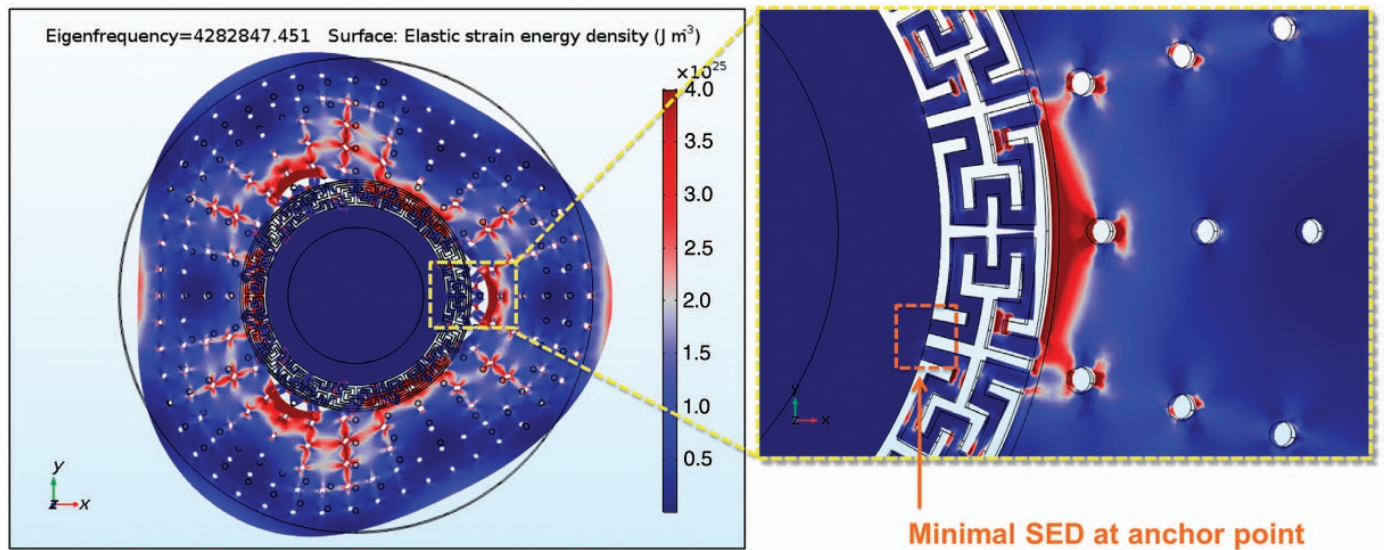

Figure 5 SED distribution for the second elliptical in-plane mode of the presented SD-BAW implemented in (100) SCS. The stress isolation system effectively attenuates the strain induced on the center support, minimizing anchor loss. BAW, bulk-acoustic wave; SCS, single-crystal silicon; SD, substrate-decoupled; SED, strain energy density.

Table 1 Quality factor for second elliptical in-plane modes

\begin{tabular}{lcc}
\hline Parameter & BAW disk resonator & SD-BAW gyroscope \\
\hline Resonance frequency, $f_{0}$ & $7.2 \mathrm{MHz}$ & $4.3 \mathrm{MHz}$ \\
Squeeze-film damping, $Q_{\text {SFD }}$ & $15000000(1 \mathrm{Torr})$ & 11000000 (1 Torr) \\
& $1500000(10 \mathrm{Torr})$ & 900000 (10 Torr) \\
Thermoelastic damping, $Q_{\text {TED }}$ & 150000 & 74000 \\
Anchor loss, $Q_{\text {anchor }}$ & 78000 & 750000000 \\
Total loss @ 10 Torr, $Q_{\text {TOTAL }}$ & 49000 & 68000 \\
\hline
\end{tabular}

BAW, bulk-acoustic wave; SD, substrate-decoupled; TED, thermoelastic damping.

isolation components are arranged in an axis-symmetric fashion (that is, similar to the release holes), both modes will experience symmetric and equal losses, minimizing damping coupling.

To quantify the level of isolation provided by the added decoupling structure, a lower bound for the anchor loss of the SD-BAW gyro can be determined using FEA simulations with perfectly matched layers (PMLs) ${ }^{26}$. As previously discussed, this method does not provide an absolute anchor-loss value but does provide an indication of the level of decoupling between a resonant structure and its substrate. This limitation exists because only a small amount of energy can be transferred from the resonator into the substrate if the worst-case $\mathcal{Q}_{\text {anchor }}$ is extremely high; therefore, the effect of any reflections will also be small.

Table 1 summarizes the simulated results for the different losses of the second elliptical in-plane modes in both a center-supported BAW disk and an SD-BAW gyroscope with the same dimensions. It is shown that for the SD-BAW gyro $\mathcal{Q}_{\text {anchor }}$ is more than four orders of magnitude larger than the dominant loss mechanism in the system $\left(\mathcal{Q}_{\text {TED }}\right)$, making its contributions to the $\mathcal{Q}_{\text {TOTAL }}$ small. Conversely, the $\mathcal{Q}_{\text {anchor }}$ of the center-support BAW disk is the dominant source of loss, making the overall system dependent on its substrate.

\section{Shock and vibration rejection}

The shock and vibration performance of a gyroscope is determined by how well the design can reject acceleration signals. In TFGs, which typically have resonance frequencies within the spectrum of environmental shock and vibration stimuli, this is typically done by using redundant proof masses that respond differentially to a Coriolis force but produce a common-mode displacement that can be rejected during linear accelerations ${ }^{32}$. However, the effectiveness of this approach depends on how well design symmetry is maintained ${ }^{14}$ and on the level of modal cross-excitation generated by errors in the capacitive sidewalls $s^{33}$. Additionally, this technique fails to reject the acceleration response of other higher-order modes that can still be within the spectrum of interest.

Conversely, the high-frequency nature of SD-BAW gyroscopes makes them inherently robust to shock and vibration. The displacement experienced by any mechanical structure in the presence of an acceleration signal $a(t)$ can be expressed in terms of the frequencies of the resonance modes with components aligned with the acceleration vector. Typically, the mode with the lowest frequency $\omega_{0 a}$ will determine the majority of the displacement $q_{\text {accel }}(t)=1 / \omega_{0 a}^{2} a(t)$. In a gyroscope, $q_{\text {accel }}(t)$ can modulate the sense mode displacement $q_{2}(t)$ if there is a residual ZRO signal due to stiffness or damping coupling, or cross-modal excitation in the case of TFGs. The acceleration-dependent ZRO can then be expressed as follows:

$$
q_{\text {2accel }}(t)=\frac{\kappa}{\omega_{0 a}^{2}} a(t) \cos (a) \sin \left(\omega_{02} t\right)
$$

where $\kappa$ is the normalized amplitude of the residual ZRO, and $a$ is the angle between the direction of the applied acceleration and the sense-mode displacement. It is worth noting that the parameter $q_{\text {accel }}(t)$ will also modulate the carrier signal of $q_{2 \text { coriolis }}(t)$ when the rotation rate is applied, even in the absence of ZRO. Therefore, reducing $K$ (that is, the ZRO amplitude) is important but not sufficient to create a gyro that is environmentally robust during operation; it is only through an increase in the value of $\omega_{0 a}$ that $q_{\text {2accel }}(t)$ can be markedly reduced. Figure 6 shows the two lowest resonance modes of the SD-BAW gyroscope. With frequencies of $200 \mathrm{kHz}$ (that is, teeter - totter mode) and $300 \mathrm{kHz}$ (that is, in-plane translational mode), these two modes are well above the frequency spectrum environmental random vibration signals, which is typically below $50 \mathrm{kHz}$.

\section{FABRICATION}

Figure 7 shows an SEM view of the fabricated SD-BAW gyroscope. Devices were fabricated using a modified version of the highaspect ratio poly- and single-crystal silicon (HARPSS ${ }^{\mathrm{TM}}$ ) process flow $^{7}$, in combination with planar $X / Y$-axis gyroscopes ${ }^{34}$ and triaxial accelerometers ${ }^{35}$ as part of a 6-degree-of-freedom system.

The process uses SOI wafers with a $40-\mu \mathrm{m}$-thick structural layer and a $2-\mu \mathrm{m}$-thick buried oxide. Lateral trenches are etched via DRIE on the device layer using a thermal-oxide mask to outline the resonator features and the surrounding electrodes (Figure 8a). A 270 -nm-thick layer of sacrificial oxide is then grown to define 

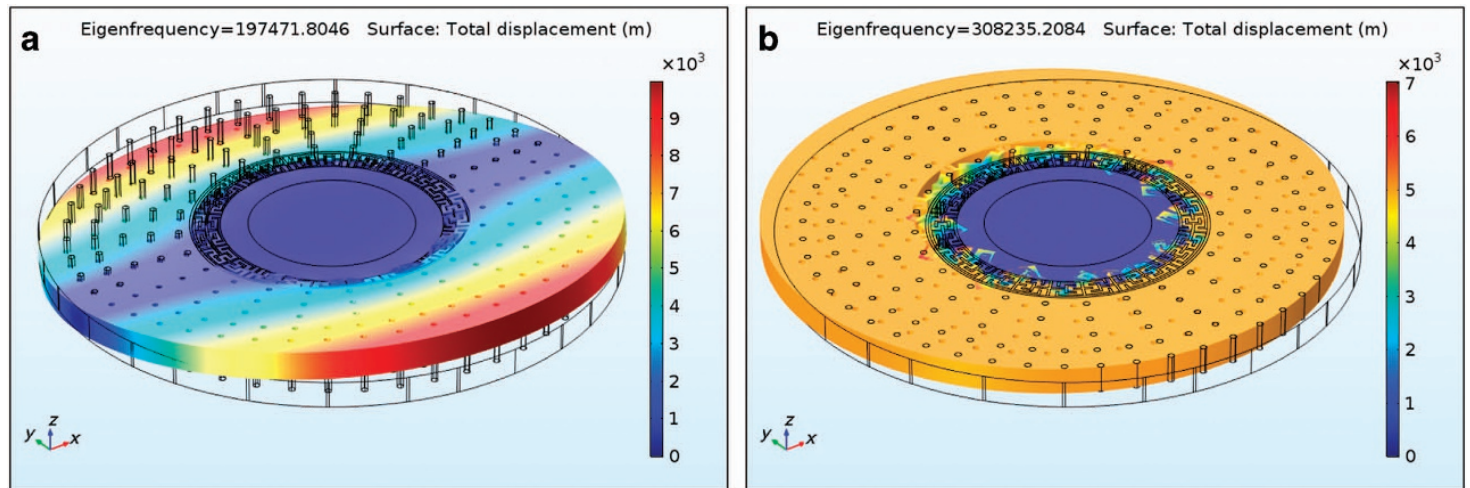

Figure 6 Simulation response for the two lowest resonance-frequency modes of an SD-BAW gyroscope. (a) The teeter - totter mode has a resonance frequency of $200 \mathrm{kHz}$, and (b) the in-plane translational mode has a resonance frequency of $300 \mathrm{kHz}$. BAW, bulk-acoustic wave; SD, substrate-decoupled.
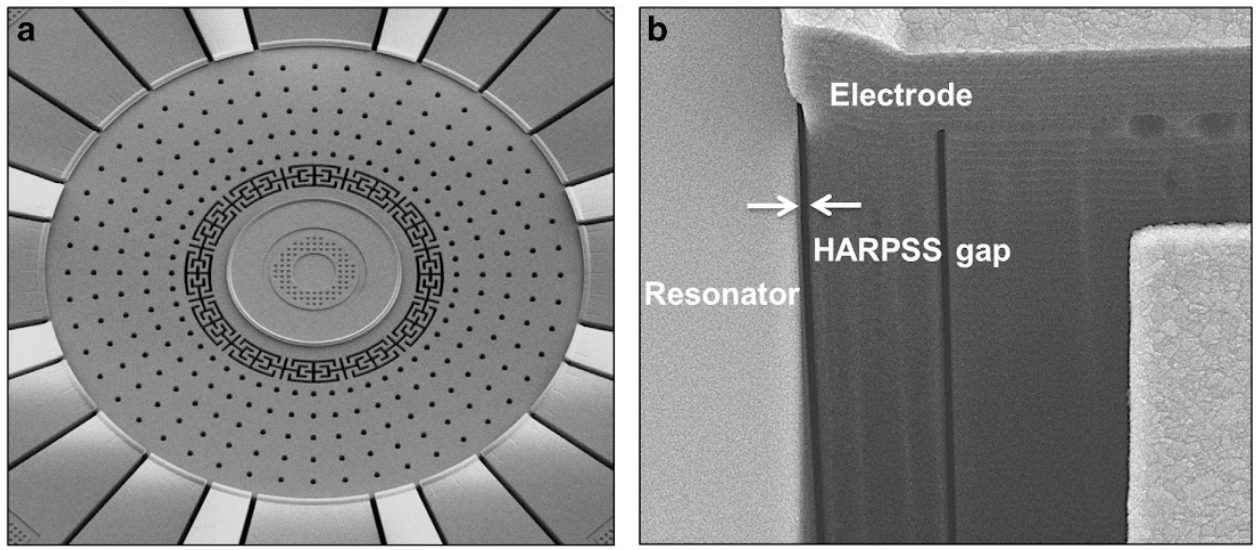

Figure 7 SEM view of the SD-BAW gyroscope (a), and close-up view of the $270 \mathrm{~nm}$ capacitive gap implemented with the HARPSSTM process (b). BAW, bulk-acoustic wave; HARPSS, high aspect-ratio combined poly and single-crystal silicon; SD, substrate-decoupled.

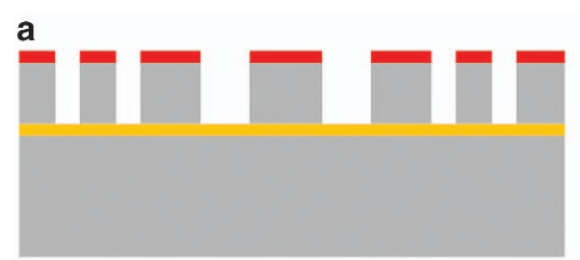

b

C

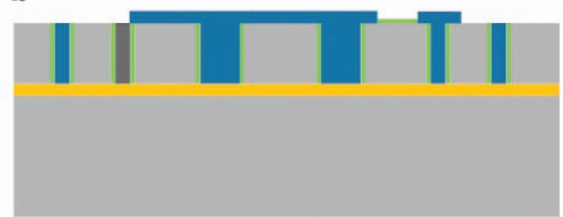

d

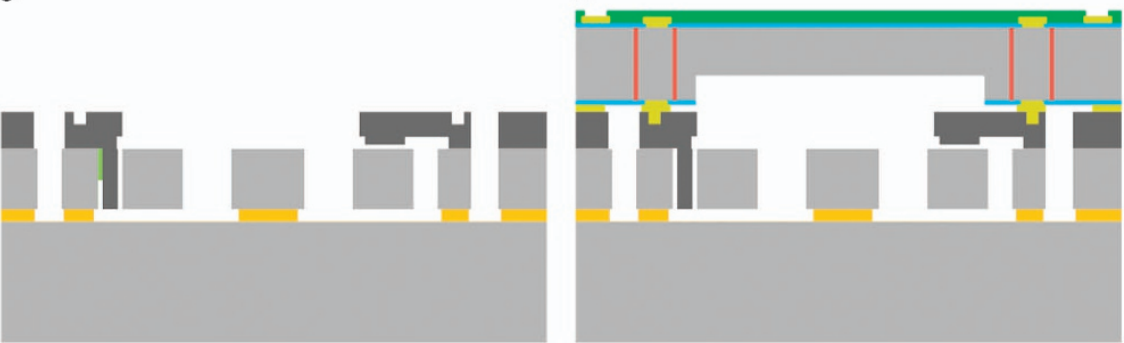

Single-crystal silicon

Thermal oxide

TEOS

Buried oxide

Sacrificial oxide for nano-gaps

Polysilicon

Figure 8 Modified process flow for the creation of SD-BAW Z-axis gyroscopes in conjunction with planar X/Y gyros and tri-axial accelerometers. (a) Structure is defined in SOI substrate via DRIE. (b) Capacitive gaps are defined by thermal oxidation, and trenches are refilled with polysilicon or TEOS to create electrodes. (c) Structure is released, and (d) device wafer is capped at a pressure of 1 to 10 Torr. BAW, bulkacoustic wave; SD, substrate-decoupled. 
a

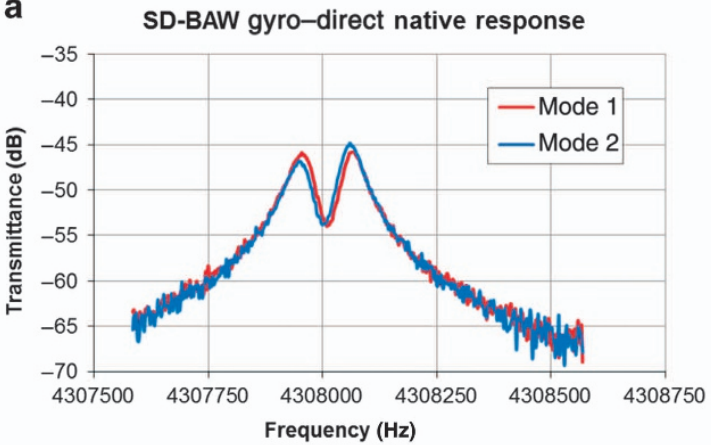

C

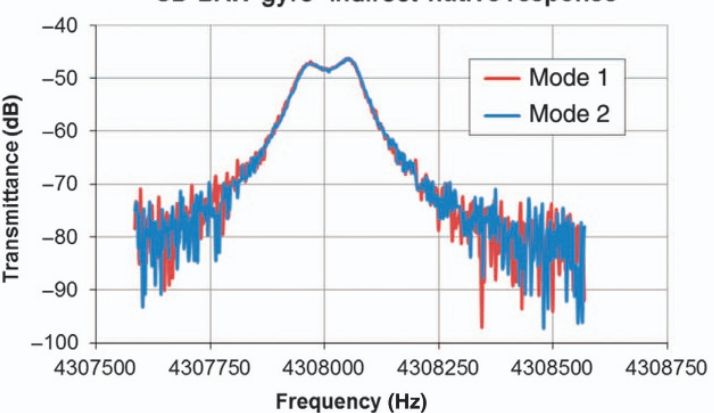

b

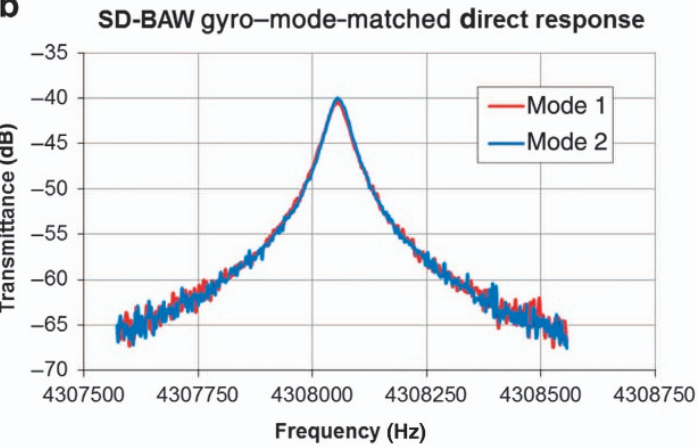

d SD-BAW gyro-mode-decoupled indirect response

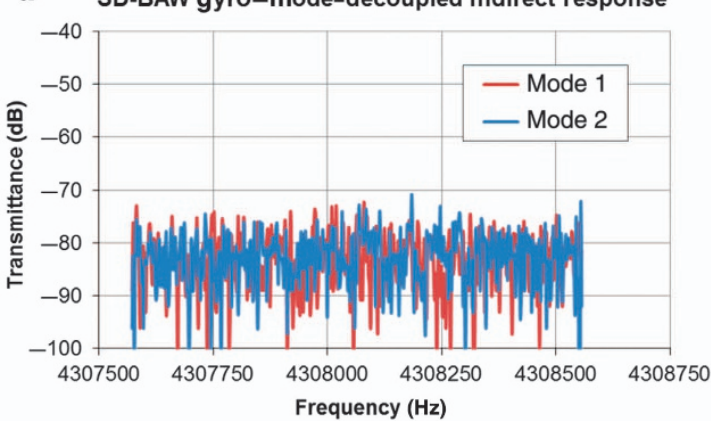

Figure 9 Frequency response of SD-BAW gyroscope at pickoff electrodes aligned directly (a, c) and orthogonal (b, d) to the mode being excited. Plots on the left are for the native response of the device, plots on the right are measured after frequency tuning and mode decoupling have occurred. BAW, bulk-acoustic wave; SD, substrate-decoupled.

the lateral (in-plane) capacitive gaps. Next, the trenches adjacent to the electrodes are re-filled with polysilicon; all other trenches are refilled with sacrificial TEOS. A second layer of sacrificial oxide ( $300 \mathrm{~nm}$ thick) is grown to define the out-of-plane capacitive gaps used for the planar gyros and accelerometers (Figure 8b). This step is followed by the deposition and patterning of polysilicon that defines the vertical electrodes. The devices are then fully released in hydrofluoric acid (HF) (Figure 8c). Lastly, a capping wafer, which is processed independently, is bonded to the base wafer to provide hermetic wafer-level packaging (WLP) at a pressure level between 1 and 10 Torr (Figure 8d). Through-silicon vias (TSVs) provide electrical connections from the device electrodes to the top of the cap wafer, and metal traces route the signals to pins at the edge of the die to facilitate wire bonding with interface electronics.

\section{CHARACTERIZATION}

\section{Standalone sensor characterization}

WLP SD-BAW gyroscopes were first characterized as standalone devices in an open-loop configuration using a network analyzer. Figure 9a shows the typical frequency response read at the electrodes aligned with the drive mode (red trace) and the sense mode (blue trace) when each mode is individually excited. The native frequency split of $110 \mathrm{~Hz}$ can be reduced down to $0 \mathrm{~Hz}$ (Figure $9 \mathrm{~b}$ ) by adjusting the tuning voltage of the mode with the lower resonance frequency. For this particular sample, $V_{\mathrm{T} 1}=3 \mathrm{~V}$ is sufficient to match the frequencies with a resonator biased at $V_{P}=18 \mathrm{~V}$ and all other electrodes at $0 \mathrm{~V}$ (Figure 9b). Conversely, Figure $9 \mathrm{c}$ shows the signal response at the electrodes aligned with the opposite mode to that being excited. The magnitude of this signal is an indication of the level of mode-to-mode coupling caused by anisoinertia and anisoelasticity. By adjusting $V_{\mathrm{Q} 1}$ to
$2.5 \mathrm{~V}$, the modes are fully decoupled (Figure 9d), showing no residual errors from damping coupling.

The mode-matched $\mathcal{Q}$ value of the WLP SD-BAW gyro is near 62000 , which is similar to the predicted $\mathcal{Q}_{\text {TOTAL }}$ value of 68000 (Table 1). To verify the effectiveness of the decoupling structure, the $\mathcal{Q}$ values of the second-elliptical modes of the gyroscope were monitored as the temperature was varied and were compared with those of a BAW disk resonator with poor substrate isolation (that is, its total $\mathcal{Q}$ is dominated by $\mathcal{Q}_{\text {anchor }}$ ). Figure 10 a clearly shows that for the SD-BAW gyro, $\mathcal{Q}$ is well behaved as the temperature changes, showing the $1 / T^{n}$ response expected by the combination of $\mathcal{Q}_{\mathrm{TED}}$ and $\mathcal{Q}_{\mathrm{SFD}}$ (in this particular case, $\left.n=1.65\right)^{36}$. Conversely, the $\mathcal{Q}$ values of the degenerate modes of a (100) SCS BAW disk resonator behave erratically over temperature, showing that the losses are highly dependent on the boundary conditions and properties of the substrate and how these vary with temperature (Figure 10b). Even more importantly, unlike the BAW disk resonator, the $\mathcal{Q}$ values for both modes of the SD-BAW gyro change identically with temperature, which guarantees that there will be no environmentally dependent damping coupling.

\section{System-level characterization}

SD-BAW gyroscopes were interfaced with an application-specific integrated circuit (ASIC) to perform a full system-level characterization of the device. The ASIC consists of a drive channel that establishes an amplitude-regulated self-oscillation loop around the drive mode and a sense channel with low-noise front-end electronics. These components are followed by an $\mathrm{I} / \mathrm{Q}$ demodulator that extracts the rotation-rate signal from its carrier and monitors the quadrature level. The quadrature signal is compared with a reference signal and is then fed back onto the mode-decoupling electrodes to maintain the gyro at an optimal operation state ${ }^{37}$. The ASIC is also equipped with a charge pump to generate the polarization and tuning voltages necessary to bias the sensor. 
a Q vs temperature of SD-BAW gyro modes

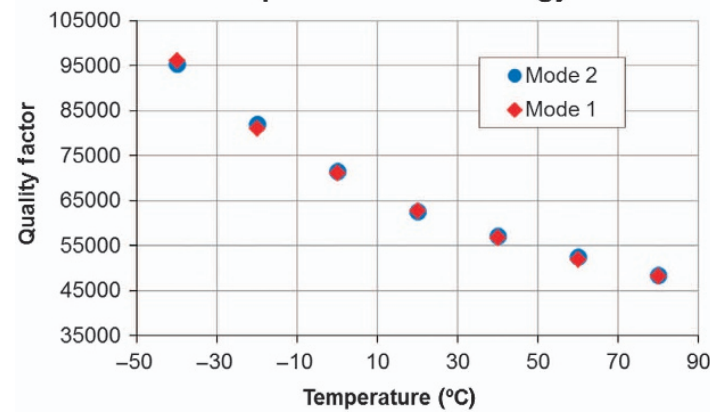

b

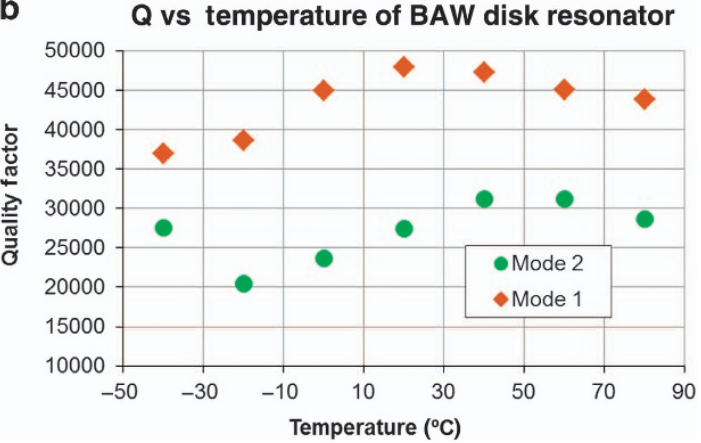

Figure 10 Quality factor variation over temperature of the second elliptical $(n=3)$ modes of an SD-BAW gyroscope (a) and a center-supported BAW disk resonator implemented on a (100) SCS substrate (b). BAW, bulk-acoustic wave; SCS, single-crystal silicon; SD, substrate-decoupled.

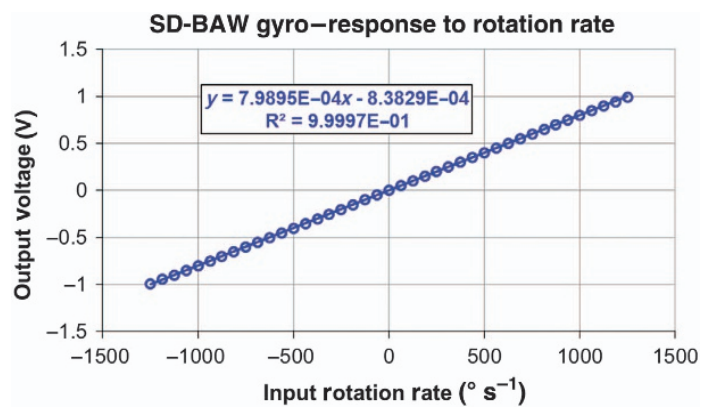

Figure 11 Output response of SD-BAW gyroscope to rotation-rate stimuli in increments of $62.5^{\circ} \mathrm{s}^{-1}$ and up to $\pm 1250^{\circ} \mathrm{s}^{-1}$. The measured non-linearity in this range is $0.07 \%$, which is limited by the interface electronics. BAW, bulk-acoustic wave; SD, substratedecoupled.

The electronic gain of the sense channel was programmed to attain an overall scale factor of $800 \mu \mathrm{V}\left({ }^{\circ} \mathrm{s}^{-1}\right)^{-1}$, which corresponds to a full-scale range of $\pm 1250^{\circ} \mathrm{s}^{-1}$ (Figure 11). The nonlinearity of the part is smaller than $0.07 \%$ and is limited by the linear range of the output buffer.

Figure 12 shows the Allan deviation plot of the SD-BAW gyroscope. The complete sensor and ASIC system exhibits an angle-random walk (ARW) of $0.39^{\circ} / \sqrt{h}\left(0.0065^{\circ} \mathrm{s}^{-1}(\sqrt{\mathrm{Hz}})^{-1}\right)$ and a bias instability $(\mathrm{BI})$ of $10.5^{\circ} \mathrm{h}^{-1}$, which are dominated by the thermal and $1 / f$ noise of the ASIC, respectively. SD-BAW devices were also interfaced with JFET-input front-end discrete electronics with reduced $1 / f$ noise and were demodulated using an HF2LI lock-in amplifier from Zurich Instruments (Zurich, Switzerland). With this configuration, the devices yielded $\mathrm{BI}$ values as low as $3.5^{\circ} \mathrm{h}^{-1}$, which are limited by a temperature-dependent feedthrough (FT) signal coupling from the drive loop to the sense channel. Further improvements of the electronics thermal noise can reduce the ARW to its theoretical limit of $0.074 \% \sqrt{h}$ (dotted trace in Figure 12), and a simple reorganization of the pin-out to minimize the FT can reduce the BI value of the system by at least 10 times.

The temperature performance of the SD-BAW gyroscope was evaluated from -40 to $85^{\circ} \mathrm{C}$. The scale factor and offset change in a typical part are shown in Figure 13. A residual change in sensitivity of $\pm 2.9 \%$ across the entire range was observed after applying a linear compensation slope to correct for the variation in $Q$ described in Figure 10a. These higher-order residuals appear due to the $1 / T^{1.65}$ temperature dependency of $Q$ described in the previous section. However, because the response is quadratic and predictable, the use of a second-order compensation scheme can reduce the scale-factor variation across the entire temperature range to less than $\pm 0.25 \%$.

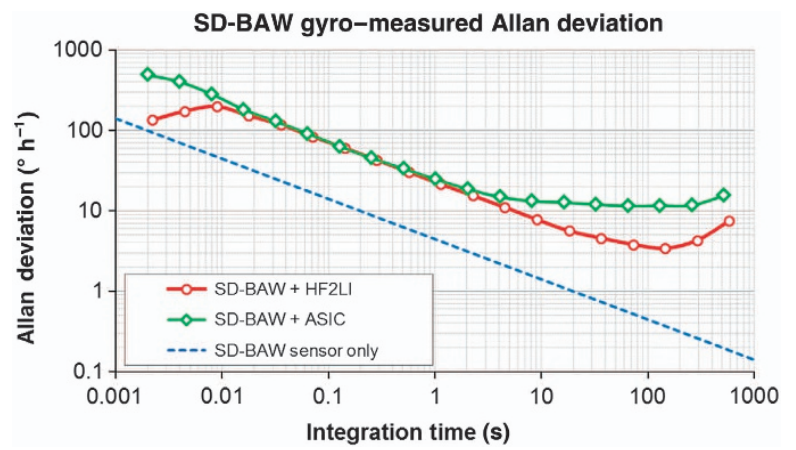

Figure 12 Allan deviation plot for SD-BAW gyroscope interfaced with customized ASIC and external electronics. The dotted trace shows the Brownian noise limit of the gyro sensor. ASIC, applicationspecific integrated circuit; BAW, bulk-acoustic wave; SD, substratedecoupled.

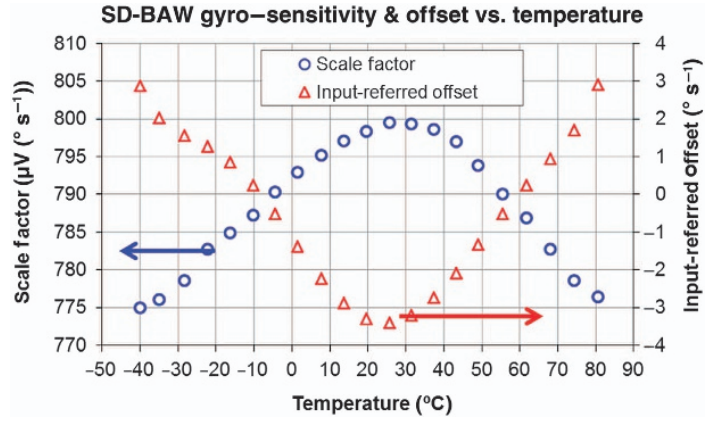

Figure 13 Variation of the scale factor and offset of an SD-BAW gyroscope as temperature varies. BAW, bulk-acoustic wave; SD, substrate-decoupled.

Conversely, the total change in the offset equals $\pm 4^{\circ} \mathrm{s}^{-1}$ across the entire temperature range and is caused by the residual temperature-dependent FT signal coupling from the drive loop to the sense channel. The quadrature control loop, which suppresses any quadrature error signal, independent of its source, will introduce an error signal in the mode-decoupling electrodes when the FT level becomes too large. This error will lead to an unwanted change in the offset. Back-end quadratic compensation could decrease this variation to $\pm 1^{\circ} \mathrm{s}^{-1}$, but the overall effect could be markedly reduced by a simple reconfiguration of the sensor pin-out to reduce the FT levels.

The robustness of the SD-BAW gyroscope with ASIC was verified by testing its immunity to random vibrations and shocks. 

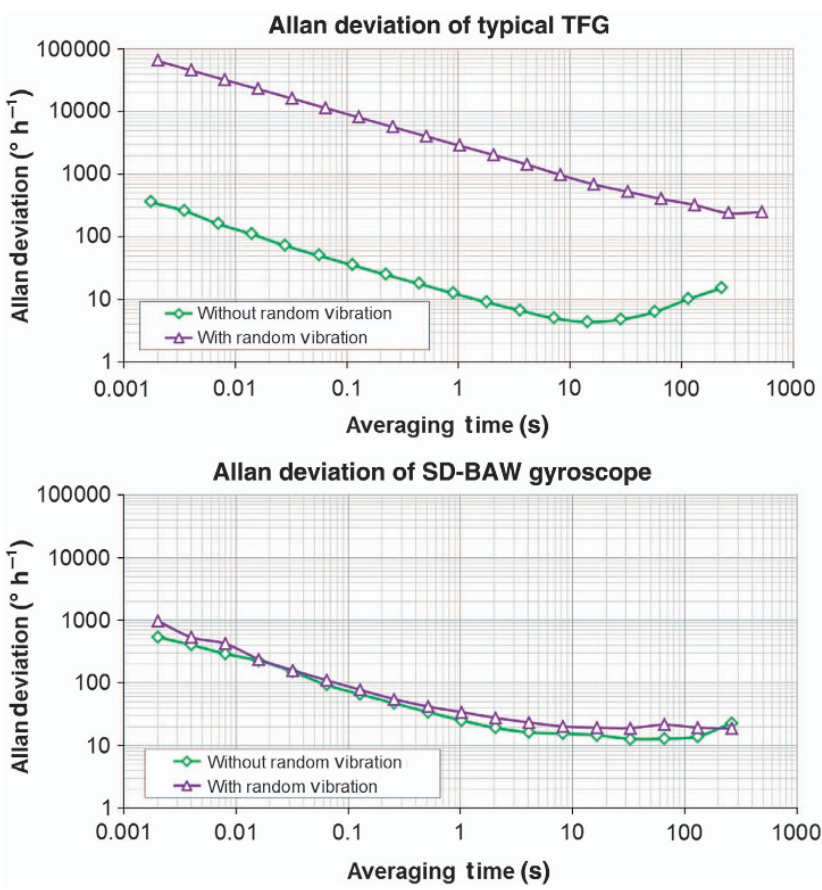

Figure 14 Change in Allan deviation response for an off-the-shelf TFG (top) and an SD-BAW gyroscope (bottom) in the presence of 14 $g_{\text {RMS }}$ of random vibration with a white frequency spectrum from $50 \mathrm{~Hz}$ to $20 \mathrm{kHz}$. BAW, bulk-acoustic wave; SD, substrate-decoupled; TFG, tuning-fork gyroscope.

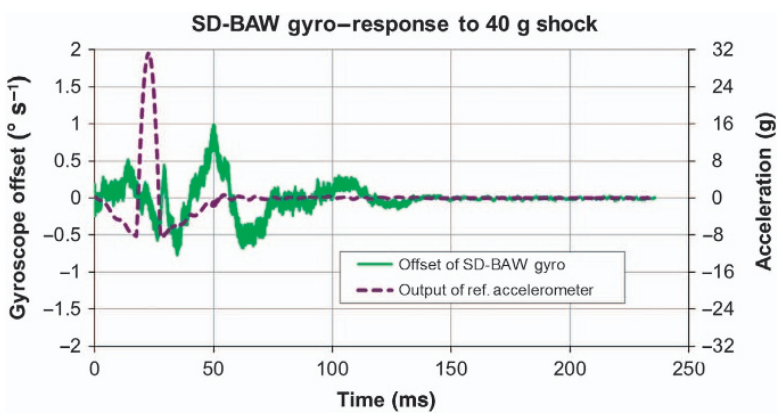

Figure 15 Maximum (peak) offset shift in the presence of a 40-g halfsine shock acceleration signal with a 10-ms duration. The dotted line represents the output of the reference accelerometer in the shock stage (right vertical axis); the continuous line represents the offset value of the gyro (left vertical axis).

Table 2 Performance metrics of the SD-BAW gyroscope

\begin{tabular}{|c|c|c|}
\hline Parameter & Value & Units \\
\hline Scale factor & 800 & $\mu \mathrm{V}\left({ }^{\circ} s^{-1}\right)^{-1}$ \\
\hline Full-scale range & \pm 1250 & ${ }^{\circ} \mathrm{s}^{-1}$ \\
\hline Non-linearity & 0.07 & $\%$ \\
\hline Angle random walk & 0.39 & $\% \sqrt{h}$ \\
\hline Bias instability & $\begin{array}{c}10.5 \text { (with ASIC) } \\
3.5 \text { (external electronics) }\end{array}$ & ${ }^{0} \mathrm{~h}^{-1}$ \\
\hline Offset over temperature & $\begin{array}{c} \pm 26 \text { (measured) } \\
\pm 6.7 \text { (with linear slope) }\end{array}$ & ${ }^{\circ} s^{-1}$ \\
\hline Random vibration sensitivity & 0.012 & ${ }^{\circ} s^{-1} g_{\text {RMS }}^{-1}$ \\
\hline Max. offset at $40 \mathrm{~g}$ shock & 1.75 & \\
\hline
\end{tabular}

ASIC, application-specific integrated circuit; BAW, bulk-acoustic wave; SD, substrate-decoupled.
The gyro was exposed to a $14-g_{\mathrm{RMS}}$ random-vibration acceleration signal with a white frequency spectrum from $50 \mathrm{~Hz}$ to $20 \mathrm{kHz}$ $\left(1 \mathrm{~g}=9.8 \mathrm{~m} \mathrm{~s}^{-1}\right)$. The measured vibration sensitivity along its most sensitive axis was as low as $0.012^{\circ} \mathrm{s}^{-1} \mathrm{~g}_{\mathrm{RM}}^{-1}$, showing the advantage of the high-frequency nature of the gyro. Figure 14 shows a comparison of the Allan deviation with and without vibration for both a commercial off-the-shelf TFG (top) and an SDBAW gyro (bottom). It is clearly shown that for the TFG, the noise performance degrades by several orders of magnitude, whereas for the SD-BAW gyro, a change in $\mathrm{BI}$ of less than $5^{\circ} \mathrm{h}^{-1}$ is observed. This result provides a clear indication of the superior noise performance of the SD-BAW gyro when operating in real environments.

The gyro system was also tested under a 40-g half-sine shock signal with a period of $10 \mathrm{~ms}$ along the most sensitive axis of the device. Figure 15 shows a maximum (that is, peak-to-peak) offset shift of only $1.75^{\circ} \mathrm{s}^{-1}$.

\section{CONCLUSION}

This paper reported the design, simulation, fabrication, and characterization of a type of high-frequency mode-matched axissymmetric gyroscope that exhibits superior environmental performance. A novel stress decoupling system is introduced to isolate the second elliptical modes of a BAW resonator from its substrate, reducing the effect of external sources of error on the sensitivity and the offset of the gyro. The current gyro performance is limited by the FT from the drive loop to the sense channel generated in the top metal traces that provide electrical connections to the electronics. Thus, further improvements in $\mathrm{BI}$ and variation of the offset and scale factor with temperature are expected through a simple reconfiguration of the pin-out. Table 2 highlights the performance metrics of the presented SD-BAW design.

\section{ACKNOWLEDGEMENTS}

The authors thank the rest of the engineering team at Qualtré for their contributions to this work.

\section{COMPETING INTERESTS}

The authors declare no conflict of interest.

\section{REFERENCES}

1 Ayazi F. Multi-DOF inertial MEMS: From gaming to dead-reckoning. The 16th International Conference on Solid-State Sensors, Actuators and Microsystems (Transducers '11); 5-9 Jun 2011; Beijing, China; 2011: 2805-2808.

2 Michalevsky Y, Boneh D, Nakibly G. Gyrophone: Recognizing speech from gyroscope signals. The 23rd USENIX Security Symposium (USENIX Security '14); $20-22$ Aug 2014; San Diego, CA, USA; 2014: 1053-1067.

3 Geen JA, Kuang J. Cross-quad and vertically coupled inertial sensors. US Patent US 7,421,897 B2, 9 Sep 2008

4 Trusov AA, Zotov SA, Shkel AM. Electrostatic regulation of quality factor in nonideal tuning fork MEMS. IEEE Sensors; 28 - 31 Oct 2011; Limerick, Ireland; 2011 20-23.

5 Johari H, Ayazi F. Capacitive bulk acoustic wave silicon disk gyroscopes. IEEE International Electron Devices Meeting (IEDM '06); 11-13 Dec 2006; San Francisco, CA, USA; 2006: 1-4.

6 Lynch D. Vibration-induced drift in the hemispherical resonator gyro. The 43rd Annual Meeting of the Institute of Navigation; 23-25 Jun 1987; Dayton, OH, USA; 1987: 26-34.

7 Ayazi F, Najafi K. High aspect-ratio combined poly and single-crystal silicon (HARPSS) MEMS technology. Journal of Microelectromechanical Systems 2000; 9: 288-294.

8 Putty MW. Micromachined vibrating ring gyroscope. PhD Dissertation, University of Michigan, 1995.

9 Bryan $\mathrm{GH}$. On the beats in the vibrations of a revolving cylinder or bell. Proceedings of the Cambridge Philosophical Society 1890; 7: 101-111. 
10 Gallacher BJ, Hedley J, Burdess JS et al. Electrostatic correction of structural imperfections present in a microring gyroscope. Journal of Microelectromechanical Systems 2005; 14: 221-234.

11 Loveday PW, Rogers CA. The influence of control system design on the performance of vibratory gyroscopes. Journal of Sound and Vibration 2002; 255: 417-432.

12 Hao Z, Pourkamali S, Ayazi. F. VHF single-crystal silicon elliptic bulk-mode capacitive disk resonators-part I: Design and modeling. Journal of Microelectromechanical Systems 2004; 13: 1043-1053.

13 Eley R, Fox CHJ, Mcwilliam S. Anisotropy effects on the vibration of circular rings made from crystalline silicon. Journal of Sound and Vibration 1999; 228: 11-35.

14 Zaman MF, Sharma A, Ayazi F. High performance matched-mode tuning fork gyroscope. The 19th IEEE International Conference on Micro Electro Mechanical Systems (MEMS '06); 22-26 Jun 2006; Istanbul, Turkey; 2006: 66-69.

15 Norouzpour-Shirazi A, Zaman MF, Ayazi F. A digital phase demodulation technique for resonant MEMS gyroscopes. IEEE Sensors Journal 2014; 14: 3260-3266.

16 Ayazi F, Sorenson L, Tabrizian R. Energy dissipation in micromechanical resonators. Proceedings of SPIE 8031, Micro- and Nanotechnology Sensors, Systems and Applications III 2011; 803119: 1-13.

17 Weinberg M, Candler R, Chandorkar S et al. Energy loss in MEMS resonators and the impact on inertial and RF devices. The 15th International Conference on SolidState Sensors Actuators and Microsystems (Transducers '09); 21 - 25 Jun 2009; Denver, CO, USA; 2009: 688-695.

18 Lynch D. Vibratory gyro analysis by the method of averaging. The 2nd St Petersburg Conference on Gyroscopic Technology and Navigation; 15-16 Sep 1995; St Petersburg, Russia; 1995: 26-34.

19 Lynch D. Coriolis Vibratory Gyros. Symposium Gyro Technology: Stuttgart, Germany. 1998: 1-14

20 Wyse SF, Stewart RE. Vibratory gyro bias error cancellation using mode reversal. US Patent US 7,886,598 B2, 15 February 2011.

21 Stewart RE. Oscillation of vibrating beam in a first direction for a first time period and a second direction for a second time period to sense angular rate of the vibrating beam. US Patent US 7,174,785 B2, 13 February 2007.

22 Sumali. H. Squeeze-film damping in the free molecular regime: model validation and measurement on a MEMS. Journal of Micromechanics and Microengineering 2007; 17: 2231-2240.

23 Zener C. Internal friction in solids II. General theory of thermoelastic internal friction. Physical Review-American Physics Society (APS) 1938; 53: 90-93.

$24 \mathrm{Hao}$ Z, Ayazi F. Support loss in the radial bulk-mode vibrations of centersupported micromechanical disk resonators. Sensors and Actuators A: Physical 2007; 134: 582-593.

25 Yang J, Ono T, Esashi M. Dominated energy dissipation in ultrathin single crystal silicon cantilever surface loss. The 13th IEEE International Conference on Micro Electro Mechanical Systems (MEMS '00); 23 - 27 Jan 2000; Miyazaki, Japan; 2000: 235-240.
26 Bindel DS, Govindjee S. Elastic PMLs for resonator anchor loss simulation. International Journal for Numerical Methods in Engineering 2005; 64: 789-818.

27 Sorenson L, Ayazi F. Effect of structural anisotropy on anchor loss mismatch and predicted case drift in future micro-hemispherical resonator gyros. The IEEE/ION Position, Location and Navigation Symposium (PLANS 2014); 5-8 May 2014; Monterey, CA, USA; 2014: 493-498.

28 Sorenson LD, Fu JL, Ayaz F. One-dimensional linear acoustic bandgap structures for performance enhancement of AIN-on-silicon micromechanical resonators. The 16th International Conference on Solid-State Sensors, Actuators and Microsystems (Transducers '11); 5 -9 Jun 2011; Beijing, China; 2011: 918-921.

29 Harrington BP, Abdolvand. R. In-plane acoustic reflectors for reducing effective anchor loss in lateral-extensional MEMS resonators. Journal of Micromechanics and Microengineering 2011; 21: 085021.

30 Wang J, Butler JE, Feygelson $\mathrm{T}$ et al. 1.51-GHz nanocrystalline diamond micromechanical disk resonator with material-mismatched isolating support. The 17th IEEE International Conference on Micro Electro Mechanical Systems (IEEE MEMS); 25-29 Jan 2004; Maastricht, The Netherlands; 2004: 641-644.

31 Lee JE-Y, Yan J, Seshia AA. Study of lateral mode SOI-MEMS resonators for reduced anchor loss. Journal of Micromechanics and Microengineering 2011; 21: 045010.

32 Trusov AA, Prikhodko IP, Zotov SA et al.. Low-dissipation silicon tuning fork gyroscopes for rate and whole angle measurements. IEEE Sensors Journal 2011; 11: $2763-2770$.

33 Weinberg MS, Kourepenis. A. Error sources in in-plane silicon tuning-fork MEMS gyroscopes. Journal of Microelectromechanical Systems 2006; 15: 479-491.

34 Sung WK. High-frequency tri-axial resonant gyroscopes. PhD Dissertation, Georgia Institute of Technology, 2013.

35 Serrano $D E$, Jeong $Y$, Keesara $V$ et al. Single proof-mass tri-axial pendulum accelerometers operating in vacuum. The 27th IEEE International Conference on Micro Electro Mechanical Systems (MEMS '14); 26 - 30 Jan 2014; San Francisco, CA, USA; 2014: 28-31.

36 Kim B, Hopcroft MA, Candler RN et al. Temperature dependence of quality factor in MEMS resonators. Journal of Microelectromechanical Systems 2008; 17: 755-766.

37 Lynch D, Savava RR, Campanile JJ et al. Hemispherical resonator gyro control. US Patent US 7,318,347 B2, 15 January 2008.

(c) (i)

This work is licensed under a Creative Commons Attribution 4.0 International License. The images or other third party material in this article are included in the article's Creative Commons license, unless indicated otherwise in the credit line; if the material is not included under the Creative Commons license, users will need to obtain permission from the license holder to reproduce the material. To view a copy of this license, visit http://creativecommons.org/licenses/ by/4.0/ 